\title{
Prevalence of Panton Valentine Leukocidin in Carriage and Infective Strains of Staphylococcus aureus at a Referral Hospital in Kenya
}

\author{
Geoffrey Omuse $^{{ }^{*}}$, Patricia Shivachi ${ }^{2}$, Samuel Kariuki ${ }^{3}$, Gunturu Revathi ${ }^{1}$ \\ ${ }^{1}$ Department of Pathology, Aga Khan University Hospital, Nairobi, Kenya \\ ${ }^{2}$ Jomo Kenyatta University of Agriculture and Technology, Nairobi, Kenya \\ ${ }^{3}$ Center of Microbiology Research, Kenya Medical Research Institute, Nairobi, Kenya \\ Email: ${ }^{*}$ g_omuse@yahoo.com
}

Received December 7, 2012; revised January 12, 2013; accepted February 1, 2013

\begin{abstract}
Panton valentine leukocidin (PVL) is a pore forming exotoxin that is expressed by some Staphylococcus aureus (S. aureus) strains and is thought to add to its virulence. The prevalence of PVL in carriage and disease causing strains varies considerably from region to region. This study compared the prevalence of the $P V L$ gene in $S$. aureus isolates obtained from healthcare workers and from patients seen at the Aga Khan University Hospital Nairobi (AKUHN). $S$. aureus isolates obtained from healthcare workers and patients attended to at AKUHN between July 2010 and March 2011 were used for this study. Forty five $S$. aureus isolates from healthcare workers and 63 from clinical specimens obtained from 59 patients were analysed for the PVL gene. The prevalence of PVL in isolates from healthcare workers was $24.4 \%$ compared to $39.7 \%$ in the isolates causing infection $(\mathrm{P}=0.098)$. $\mathrm{PVL}$ prevalence was $58.8 \%$ in $S$. aureus isolates obtained from skin and soft tissue infections (SSIs) compared to $25.0 \%$ in carriage isolates ( $\mathrm{P}=0.002$, OR 4.29). Prevalence in isolates from invasive infections was $11.1 \%$. Patients with PVL positive S. aureus were younger than those with PVL negative isolates $(\mathrm{P}=0.082)$. The high prevalence of PVL is comparable with that reported in other African countries. The significance of the high prevalence of PVL in S.aureus isolates carried by health care workers at AKUHN is unclear at the moment. PVL prevalence is significantly higher in S. aureus isolates causing SSIs compared to carriage and invasive isolates.
\end{abstract}

Keywords: Staphylococcus aureus; S. aureus; Panton Valentine Leukocidin; PVL

\section{Introduction}

Panton valentine leukocidin (PVL) is a pore forming exotoxin that is expressed by some Staphylococcus aureus (S. aureus) strains. It was named after Sir Philip Noel Panton and Francis Valentine when they associated it with soft tissue infections in 1932 [1]. It is composed of two water soluble proteins LukF-PV and LukS-PV that specifically lyse polymorphonuclear cells and monocytes by creating pores in their cell membranes [2].

S. aureus strains producing PVL have been associated with a variety of illnesses ranging from skin and soft tissue infections to serious infections like necrotizing pneumonia that are invariably fatal [3]. Unfortunately, the necrotizing pneumonia caused by these strains is more common in young healthy adults and mortality is usually very high despite treatment [4]. Studies carried out on rabbit models have shown that the increased lung

\footnotetext{
${ }^{*}$ Corresponding author.
}

damage caused by PVL positive strains is mediated by toxic granules released by polymorphonuclear cells due to PVL mediated cytolysis. However, lung damage can also occur in the absence of PVL but the morbidity is less [5].

The prevalence of PVL in S. aureus isolates obtained from clinical specimens has been found to vary significantly from country to country with prevalence as high as $57 \%$ seen in isolates from west African countries compared to $9.7 \%$ in England [6,7]. Whether the presence of PVL translates to more severe disease in humans is debatable however they are associated with recurrent soft tissue infections. An observational study carried out in New Zealand found that patients with PVL-positive methicillin susceptible S. aureus (MSSA) Surgical Site Infections were 7.4 times more likely to require surgical drainage of cutaneous abscesses compared to patients with PVL negative infections [8]. However, some experimental and epidemiologic studies have shown that PVL may not be a major virulence factor in causing 
community acquired methicillin resistant $S$. aureus infections (MRSA) [9,10]. Bae et al. showed that factors other than the presence of PVL are the primary determinants of clinical outcome in complicated skin and skin structure infections caused by MRSA [11]. Some of the in-vitro studies that have questioned the role of PVL in pathogenesis were based on murine models whose neutrophils have been shown to be inherently resistant to PVL. PVL has been shown to act differentially on neutrophils from various animal species and as such careful selection of animal models is required to aid in properly defining the pathological role of PVL. In this regard, rabbit models have been found to be the best human surrogates $[12$, 13].

Few studies have attempted to compare the prevalence of $P V L$ in colonizing and disease causing strains of $S$. aureus [14-16]. We hypothesized that the prevalence of $P V L$ would be higher in S. aureus isolates causing infection compared to carriage isolates given the evidence of increased virulence conferred by PVL $[8,17]$. This study compared the prevalence of the $P V L$ gene in $S$. aureus isolates obtained from the anterior nares of healthcare workers and S. aureus isolates from patients seen at the Aga Khan University Hospital Nairobi (AKUHN).

\section{Methodology}

AKUHN is a 254-bed private referral hospital with satellite clinics and laboratories serving a sizeable population in Nairobi and other major towns in Kenya. The hospital has all major medical disciplines including a state of the art heart and cancer centre, dialysis units, adult and paediatric high dependency and intensive care units. The laboratory at the main hospital has Microbiology, Haematology, Clinical chemistry and Anatomical pathology sections that are all ISO 15189:2007 accredited by the South African National Accreditation System (SANAS).

\subsection{Samples}

S. aureus isolates obtained from healthcare workers and patients attended to at AKUHN were used for the study. The isolates from healthcare workers were obtained as part of a previous study looking at nasal carriage of MRSA at AKUHN between July and December 2010. Two hundred and sixty one healthcare workers were selected at random from all clinical departments using a predefined procedure. The departments included pathology, radiology, accident and emergency, paediatrics, surgery, obstetrics and gynaecology, adult medical wards, intensive care and high dependency units, physiotherapy and operating theatres. Participation was voluntary and written informed consent was obtained [18]. S. aureus isolates from patients were obtained sequentially from clinical specimens submitted to the Microbiology divi- sion of the Pathology department for routine diagnostic and surveillance cultures between January and March 2011. These included blood, urine, pus swabs, surveillance swabs (nasal, axillary and groin) and body fluids. Bacterial isolates were frozen in brain heart infusion with $10 \%$ glycerol at $-80^{\circ} \mathrm{C}$ until analysis.

\subsection{Identification of $S$. aureus, MecA and $P V L$ Genes}

Frozen isolates were thawed at room temperature and cultured on to blood agar plates (Oxoid Ltd., Basingstoke, UK). Colonies morphologically resembling S. aureus were identified using standard bench methods which included gram stain, catalase test, tube and slide coagulase tests. Confirmed $S$. aureus strains were subjected to disc diffusion using $1 \mu \mathrm{g}$ oxacillin and $30 \mu \mathrm{g}$ cefoxitin discs (BD BBL, MD, USA) to identify MRSA. Interpretation of the tests was based on Clinical Laboratory Standards Institute guidelines [19]. Genomic DNA was extracted from all the S. aureus isolates using QIAamp DNA Mini Kit (QIAGEN, Valencia, CA, USA) according to the manufacturer's instructions. All S. aureus isolates were then tested for the presence of MecA and PVL genes using the Genotype MRSA kit version 2.0 (Hain Lifescience, Nehren, Germany). Appropriate controls were included for all tests.

\subsection{Ethical Approval}

Ethical approval was obtained from the AKUHN scientific and ethics committees.

\subsection{Statistics}

Descriptive statistics were presented as means or proportions as appropriate. The Kolmogorov Smirnoff test was used to test for normality of data. All inferential statistics were exploratory. The independent sample t-test was used to compare means for parametric data while the Mann Whitney U test was used for non-parametric data. Chisquare test was used to compare proportions. All tests were two sided. A p value less than 0.05 was considered statistically significant. Statistical analysis was carried out using SPSS version 15.0 (SPSS, Inc., Chicago IL).

\section{Results}

Forty five S. aureus isolates from healthcare workers and 63 from clinical specimens obtained from 59 patients were analysed for the presence of the $P V L$ gene. The mean (SD) age in years for the healthcare workers and patients was 34.5 (6.7) and 34.3 (22.1) respectively. The difference was not statistically significant $(t(71.6)=$ $0.078, \mathrm{P}=0.938)$. There were $27(60 \%)$ and $37(62.7 \%)$ males among healthcare workers and patients respec- 
tively.

The prevalence of $P V L$ in the isolates obtained from healthcare workers was $24.4 \%(11 / 45)$ compared to $39.7 \%(25 / 63)$ in the isolates obtained from patients $(\mathrm{P}=$ $0.098)$. The mean age for patients whose specimens had $P V L$ positive $S$. aureus was significantly lower than those with $P V L$ negative $S$. aureus with mean (SD) ages in years of 25.7 (17.1) and 42.4 (23.2) respectively $(U=$ $271, \mathrm{P}=0.004)$. Amongst health care workers, a similar difference in age was not seen between carriers of $P V L$ positive and $P V L$ negative $S$. aureus. Their mean ages (SD) in years were 35 (6.5) and 34.4 (6.9) respectively $(U=163.5, \mathrm{P}=0.540)$.

The prevalence of $P V L$ in the different specimen types is shown in Table 1. Pus swabs had the highest prevalence of $57.1 \%(20 / 35)$ and of the 10 blood specimens, only 1 had $P V L$. The prevalence of $P V L$ in carriage strains obtained from both healthcare workers and patients was $25.0 \%$ compared to $42.3 \%(22 / 52)$ in disease causing strains. This difference wasn't statistically significant ( $\mathrm{P}=0.059$, OR 2.2; 95\% CI: 0.97 - 4.98). However, a comparison between $P V L$ prevalence in $S$. aureus isolates from SSIs and those from non SSIs (4 urine, 10 blood, 2 ascitic fluid, 1 pus swab and 1 sputum) showed a significant difference with the prevalence being higher in SSIs ( $\mathrm{P}=0.003$, OR 11.43; 95\% CI: 2.26 - 57.8). A similar comparison between SSI isolates and screening swabs (45 nasal swabs from healthcare workers and 11 surveillance swabs from patients) also showed a significant difference with a higher prevalence in SSIs $(\mathrm{P}=$ 0.002, OR 4.29; 95\% CI: 1.72 - 10.67).

Table 2 shows the prevalence of PVL in S.aureus isolates from SSIs, non SSIs and screening swabs. The mean (SD) age for patients with SSIs was 30.4 (22) years while that for patients with invasive infections was

Table 1. Prevalence of $P V L$ gene in S. aureus isolates obtained from different patient specimens.

\begin{tabular}{cccc}
\hline \multirow{2}{*}{ Specimen type } & \multicolumn{2}{c}{ PVL gene number (percentage) } & \multirow{2}{*}{ Total } \\
\cline { 2 - 3 } & Absent & Present & \\
\hline Urine & $4(100 \%)$ & $0(0 \%)$ & 4 \\
Pus swab & $15(42.9 \%)$ & $20(57.1 \%)$ & 35 \\
Blood & $9(90 \%)$ & $1(10 \%)$ & 10 \\
Surveillance swab & $8(72.7 \%)$ & $3(27.3 \%)$ & 11 \\
Ascitic fluid & $1(50 \%)$ & $1(50 \%)$ & 2 \\
Sputum & $1(100 \%)$ & $0(0 \%)$ & 1 \\
Total & $38(60.3 \%)$ & $25(39.7 \%)$ & 63 \\
\hline
\end{tabular}

${ }^{a}$ Surveillance swabs include nasal, axillary and groin swabs collected from patients as part of screening on admission into the intensive care and high dependency units.
Table 2. Prevalence of $P V L$ in colonizing and disease causing strains of $S$. aureus.

\begin{tabular}{cccc}
\hline \multirow{2}{*}{ Specimen type } & \multicolumn{2}{c}{$\begin{array}{c}P V L \text { gene: number } \\
\text { (percentage) }\end{array}$} & \multirow{2}{*}{ Total } \\
\cline { 2 - 3 } & Absent & Present & \\
\hline Skin and soft tissue $^{\mathrm{a}}$ & $14(41.2 \%)$ & $20(58.8 \%)$ & 34 \\
Non skin and soft tissue $^{\mathrm{b}}$ & $16(88.2 \%)$ & $2(11.1 \%)$ & 18 \\
Screening swab $^{\mathrm{c}}$ & $42(75.0 \%)$ & $14(25.0 \%)$ & 56 \\
Total & $74(63.8 \%)$ & $42(36.2 \%)$ & 108 \\
\hline
\end{tabular}

${ }^{\mathrm{a}}$ Includes all pus swabs obtained from skin and soft tissue infections (SSIs); bIncludes urine, blood, sputum, pus and ascitic fluid representing invasive infections; ' Includes nasal swabs from healthcare workers and surveillance swabs (nasal, axillary and groin) from patients.

41.2 (24.3) years. This difference wasn't statistically significant $(U=208.5, \mathrm{P}=0.082)$.

Only 9 of the $63(14.3 \%)$ S. aureus isolates obtained from the patient specimens had the MecA gene detected. Of these, 3 also had the PVL gene. Two of the isolates were from pus swabs and the other was from ascitic fluid.

\section{Discussion}

The precise role of single virulence factors in the causation of infectious disease is hard to establish and PVL is no exception. Several studies do suggest that PVL is one of the major virulence factors contributing to the morbidity and mortality attributed to $S$. aureus [3,4,17]. This contribution has however been put to question by studies that have shown a better clinical outcome for patients with SSIs caused by PVL producing MRSA compared to strains not producing PVL $[11,20]$. Otto et al. have suggested that factors other than the mere presence of PVL are the primary determinants of clinical outcome in patients with S. aureus SSIs [21]. This study found the prevalence of $P V L$ in $S$. aureus isolates causing infection to be $42.3 \%$. Compared to the prevalence in carriage strains, the difference wasn't statistically significant but tended towards significance with a $\mathrm{P}$ value of 0.059 . Feil et al. showed clonal similarity between carrier and disease causing strains of $S$. aureus from a localized population in Oxfordshire, United Kingdom suggesting that $S$. aureus infections are opportunistic in nature and as such cases of disease are thought to represent a random sample of the much larger reservoir of carriage strains. No significant differences should therefore exist between carriage and disease causing strains of S. aureus [22]. In this study, a comparison between the prevalence of the $P V L$ gene in $S$. aureus isolates from patients with SSIs and those with non SSIs showed a significantly higher prevalence in the former. This finding is in keeping with what has been previously documented. Prevost et al. 
found that $23.6 \%$ of $S$. aureus isolates from cutaneous infections had the PVL gene compared to $1.4 \%$ of blood isolates [23]. A similar finding was seen in Gabon where the prevalence of $P V L$ in isolates from abscesses was $100 \%$ while in blood isolates it was $54.5 \%$. Despite similarities in clonality as determined by multilocus sequence typing (MLST) between carriage and disease causing strains of $S$. aureus, true differences may actually exist. The apparent clonal similarity between carriage and invasive S. aureus strains as reported by Feil et al. may be as a result of limitations of the MLST typing method used which only looks at housekeeping genes [24]. It is therefore possible that there could be differences in the population structure of $S$. aureus based on variations in accessory genes that MLST could miss. The differential occurrence of the PVL gene in S. aureus isolates causing SSIs and non SSIs in this study may be a reflection of such variability. PVL producing $S$. aureus appear to be successful pathogens with a preference for SSIs as seen in this study.

Nasal carriage of $S$. aureus by healthcare workers at AKUHN has previously been reported to be $18.3 \%$ while MRSA was found to be absent [18]. The isolates obtained during the carriage study were used in this study to determine $P V L$ gene prevalence in carriage strains of S.aureus which was found to be $24.4 \%$. This is comparable with prevalence found in other African countries which ranges between $17 \%$ and $74 \%$ but is considerably higher than that found in other continents where the prevalence of $\mathrm{PVL}$ in carriage isolates was found to range from $0 \%$ to $10.6 \%[6,14-16,25-27]$. It is possible that there is a $S$. aureus clone harboring the PVL gene circulating amongst healthcare workers at AKUHN hence the high prevalence. However, PVL is commonly associated with community acquired isolates and the high prevalence could indeed be a true reflection of the status quo as seen in the other African studies [28]. In Mali, a pandemic clone (ST 152) that comprised $23.9 \%$ of carriage isolates was identified and all the isolates belonging to this clone were found to harbour the PVL gene [29]. The carriage isolates in the Mali study were obtained from patients admitted for emergency surgery and most likely represent community isolates. The prevalence of $P V L$ in $S$. aureus isolates obtained from a healthy non hospital based population in the community served by AKUHN may help in better understanding the epidemicology of these isolates. The significance of the high prevalence of $P V L$ in S. aureus isolates carried by healthcare workers at AKUHN is unclear at the moment. Comparative genomics of the carriage and disease causing strains may help ascertain the disease causing potential of the carriage strains by determining clonality.

Only 9 S. aureus isolates from clinical specimens were found to possess the MecA gene and of these 3 had the
$P V L$ gene. These 3 isolates may be community acquired MRSA given that PVL presence is more common in community acquired isolates [30]. There is a paucity of published data on community acquired MRSA (CAMRSA) isolates harbouring the $P V L$ gene from East Africa. The emergence and spread of such isolates has already been reported in Algeria, Cameroon, Morocco, Nigeria, and Senegal $[6,31,32]$. Globally, CA-MRSA has been the source of numerous outbreaks causing significant morbidity and mortality [33-35]. Fortunately, none of the carriage S.aureus isolates from health care workers at AKUHN harboured both the MecA and PVL genes. However, such strains could still make their way from the community to the hospital like the USA 300 strain, a PVL producing CA-MRSA, which has begun to displace traditional hospital acquired MRSA strains in the United States $[34,36]$. Continuous monitoring of microbiological data on both community and hospital acquired infections and appropriate containment measures will go a long way to ensure that PVL producing CA-MRSA doesn't become endemic at AKUHN.

This study found that patients with $P V L$ positive $S$. aureus were younger than patients with $P V L$ negative strains. This finding is not unique to this study. Tong et al analysed data from two large, multinational phase three clinical trials assessing ceftaroline fosamil for the treatment of complicated skin and skin structure related infections and found that infections caused by $P V L$ positive $S$. aureus were associated with a younger patient age for both MRSA and MSSA [37]. We hypothesize that this finding may be an affirmation of the increased virulence of PVL producing S.aureus. Host immune response is one function of the body profoundly affected by aging due to "immunosenescence" [38]. Younger adults can be assumed to be more immunocompetent compared to an older population. It would therefore take a more virulent strain of $S$. aureus to cause disease in this population. Tong et al. found that patients with $P V L$ negative strains tended to be older and had comorbidities such as diabetes or peripheral vascular disease [37]. This group of patients is likely to have reduced host immunity and would be prone to infection even by a less virulent strain of S. aureus. The finding by Tong et al. that PVL is not the primary determinant in SSIs may therefore be confounded by the occurrence of PVL positive S. aureus infections in a younger population who can mount a robust immune response. Comparison of clinical outcomes in patients matched for age and comorbidities would help clarify the role of PVL in determining clinical outcomes.

A major limitation of this study is the relatively few numbers of $S$. aureus isolates studied especially from clinical specimens. The results of associations tested are therefore exploratory and would best be re-tested in a study powered to test these associations. However, some 
of the findings are consistent with what larger studies have found.

\section{Conclusion}

A study specifically powered to show a difference in $P V L$ prevalence between carriage and infective strains of $S$. aureus is required. Further studies are also required to determine the significance of the high prevalence of $P V L$ in $S$. aureus isolates carried by healthcare workers at AKUHN and whether they contribute to hospital acquired $S$. aureus infections. The isolation of a few $P V L$ positive MRSA isolates from clinical specimens' raises concern given their increased virulence and multidrug resistant nature as epitomized by the USA 300 strain. Continuous surveillance and appropriate infection control measures are necessary to ensure that such isolates don't become endemic in the hospital. More studies are required to define the population structure and dynamics of $S$. aureus carriage and infective strains from the region.

\section{Acknowledgements}

Aga Khan University Hospital Nairobi for providing funds to buy most of the materials used in the study. Ms. Alice Gichane of Hain Life science, Nairobi, Kenya for providing two free Genotype MRSA kits version 2.0 (Hain Lifescience, Nehren, Germany).

\section{REFERENCES}

[1] P. Panton and F. Valentine, "Staphylococcal Toxin," Lancet, Vol. 219, No. 5662, 1932, pp. 506-508. doi:10.1016/S0140-6736(01)24468-7

[2] T. Tomita and Y. Kamio, "Molecular Biology of the Pore-Forming Cytolysins from Staphylococcus aureus, Alpha- and Gamma-Hemolysins and Leukocidin," Bioscience, Biotechnology, and Biochemistry, Vol. 61, No. 4, 1997, pp. 565-572. doi:10.1271/bbb.61.565

[3] G. Lina, Y. Piemont, F. Godail-Gamot, M. Bes, M. O. Peter, V. Gauduchon, et al., "Involvement of PantonValentine Leukocidin-Producing Staphylococcus aureus in Primary Skin Infections and Pneumonia," Clinical Infectious Diseases, Vol. 29, No. 5, 1999, pp. 1128-1132. doi:10.1086/313461

[4] Y. Gillet, B. Issartel, P. Vanhems, J. C. Fournet, G. Lina, M. Bes, et al., "Association between Staphylococcus aureus Strains Carrying Gene for Panton-Valentine Leukocidin and Highly Lethal Necrotising Pneumonia in Young Immunocompetent Patients," Lancet, Vol. 359, No. 9308, 2002, pp. 753-759. doi:10.1016/S0140-6736(02)07877-7

[5] B. A. Diep, L. Chan, P. Tattevin, O. Kajikawa, T. R. Martin, L. Basuino, et al., "Polymorphonuclear Leukocytes Mediate Staphylococcus aureus Panton-Valentine Leukocidin-Induced Lung Inflammation and Injury," Proceedings of the National Academy of Sciences of USA, Vol. 107, No. 12, 2010, pp. 5587-5592 doi:10.1073/pnas.0912403107

[6] S. Breurec, C. Fall, R. Pouillot, P. Boisier, S. Brisse, F. Diene-Sarr, et al., "Epidemiology of Methicillin-Susceptible Staphylococcus aureus Lineages in Five Major African Towns: High Prevalence of Panton-Valentine Leukocidin Genes," Clinical Microbiology and Infection, Vol. 17, No. 4, 2010, pp. 633-639. doi:10.1111/j.1469-0691.2010.03320.x

[7] L. J. Shallcross, K. Williams, S. Hopkins, R. W. Aldridge, A. M. Johnson and A. C. Hayward, "Panton Valentine Leukocidin Associated Staphylococcal Disease: A Cross Sectional Study at a London Hospital, England," Clinical Microbiology and Infection, Vol. 16, No. 11, 2010, pp. 1644-1648. doi:10.1111/j.1469-0691.2010.03153.x

[8] S. Muttaiyah, G. Coombs, S. Pandey, P. Reed, S. Ritchie, D. Lennon, et al., "Incidence, Risk Factors, and Outcomes of Panton-Valentine Leukocidin-Positive Methicillin-Susceptible Staphylococcus aureus Infections in Auckland, New Zealand," Journal of Clinical Microbiology, Vol. 48, No. 10, 2010, pp. 3470-3474. doi:10.1128/JCM.00911-10

[9] J. M. Voyich, M. Otto, B. Mathema, K. R. Braughton, A. R. Whitney, D. Welty, et al., "Is Panton-Valentine Leukocidin the Major Virulence Determinant in CommunityAssociated Methicillin-Resistant Staphylococcus aureus Disease?" The Journal of Infectious Diseases, Vol. 194, No. 12, 2006, pp. 1761-1770. doi:10.1086/509506

[10] J. B. Wardenburg, A. M. Palazzolo-Ballance, M. Otto, O. Schneewind and F. R. DeLeo, "Panton-Valentine Leukocidin Is Not a Virulence Determinant in Murine Models of Community-Associated Methicillin-Resistant Staphylococcus aureus Disease," The Journal of Infectious Diseases, Vol. 198, No. 8, 2008, pp. 1166-1170. doi:10.1086/592053

[11] I. G. Bae, G. T. Tonthat, M. E. Stryjewski, T. H. Rude, L. F. Reilly, S. L. Barriere, et al., "Presence of Genes Encoding the Panton-Valentine Leukocidin Exotoxin Is Not the Primary Determinant of Outcome in Patients with Complicated Skin and Skin Structure Infections Due to Methicillin-Resistant Staphylococcus aureus: Results of a Multinational Trial," Journal of Clinical Microbiology, Vol. 47, No. 12, 2009, pp. 3952-3957. doi:10.1128/JCM.01643-09

[12] U. Lipinska, K. Hermans, L. Meulemans, O. Dumitrescu, C. Badiou, L. Duchateau, et al., "Panton-Valentine Leukocidin Does Play a Role in the Early Stage of Staphylococcus aureus Skin Infections: A Rabbit Model," PLoS One, Vol. 6, No. 8, 2011, e22864. doi:10.1371/journal.pone.0022864

[13] B. Loffler, M. Hussain, M. Grundmeier, M. Bruck, D. Holzinger, G. Varga, et al., "Staphylococcus aureus Panton-Valentine Leukocidin Is a Very Potent Cytotoxic Factor for Human Neutrophils," PLoS Pathogens, Vol. 6, No. 1, 2010, e1000715. doi:10.1371/journal.ppat.1000715

[14] D. C. Melles, W. B. van Leeuwen, H. A. Boelens, J. K. Peeters, H. A. Verbrugh and A. van Belkum, "PantonValentine Leukocidin Genes in Staphylococcus aureus," Emerging Infectious Diseases, Vol. 12, No. 7, 2006, pp. 1174-1175. doi:10.3201/eid1207.050865

[15] V. Neela, G. R. Ehsanollah, S. Zamberi, A. Van Belkum 
and N. S. Mariana, "Prevalence of Panton-Valentine Leukocidin Genes among Carriage and Invasive Staphylococcus aureus Isolates in Malaysia," International Journal of Infectious Diseases, Vol. 13, No. 3, 2009, pp. e131e132. doi:10.1016/j.ijid.2008.07.009

[16] F. Schaumburg, U. A. Ngoa, K. Kosters, R. Kock, A. A. Adegnika, P. G. Kremsner, et al., "Virulence Factors and Genotypes of Staphylococcus aureus from Infection and Carriage in Gabon," Clinical Microbiology and Infection, Vol. 17, No. 10, 2011, pp. 1507-1513. doi:10.1111/j.1469-0691.2011.03534.x

[17] Y. Gillet, J. Etienne, G. Lina and F. Vandenesch, "Association of Necrotizing Pneumonia with Panton-Valentine Leukocidin-Producing Staphylococcus aureus, Regardless of Methicillin Resistance," Clinical Infectious Disease, Vol. 47, No. 7, 2008, pp. 985-986. doi: $10.1086 / 591803$

[18] G. Omuse, S. Kariuki and G. Revathi, "Unexpected Absence of Meticillin-Resistant Staphylococcus aureus Nasal Carriage by Healthcare Workers in a Tertiary Hospital in Kenya," Journal of Hospital Infection, Vol. 80, No. 1, 2011, pp. 71-73. doi:10.1016/j.jhin.2011.09.009

[19] Clinical and Laboratory Standards Institute, "Performance Standards for Antimicrobial Susceptibility Testing," 20th Informational Supplement, CLSI, Vol. 29, M100-S20, 2010.

[20] S. J. Campbell, H. S. Deshmukh, C. L. Nelson, I. G. Bae, M. E. Stryjewski, J. J. Federspiel, et al., "Genotypic Characteristics of Staphylococcus aureus Isolates from a Multinational Trial of Complicated Skin and Skin Structure Infections," Journal of Clinical Microbiology, Vol. 46, No. 2, 2008, pp. 678-684. doi:10.1128/JCM.01822-07

[21] M. Otto, “A MRSA-Terious Enemy among Us: End of the PVL Controversy?" Nature Medicine, Vol. 17, 2011, pp. 169-170. doi:10.1038/nm0211-169

[22] E. J. Feil, J. E. Cooper, H. Grundmann, D. A. Robinson, M. C. Enright, T. Berendt, et al., "How Clonal Is Staphylococcus aureus?” Journal of Bacteriology, Vol. 185, No. 11, 2003, pp. 3307-3316. doi:10.1128/JB.185.11.3307-3316.2003

[23] G. Prevost, P. Couppie, P. Prevost, S. Gayet, P. Petiau, B. Cribier, et al., "Epidemiological Data on Staphylococcus aureus Strains Producing Synergohymenotropic Toxins," Journal of Medical Microbiology, Vol. 42, No. 4, 1995, pp. 237-245. doi:10.1099/00222615-42-4-237

[24] T. Ferry, D. Thomas, A. L. Genestier, M. Bes, G. Lina, F. Vandenesch, et al., "Comparative Prevalence of Superantigen Genes in Staphylococcus aureus Isolates Causing Sepsis with and without Septic Shock," Clinical Infectious Diseases, Vol. 41, No. 6, 2005, pp. 771-777. doi:10.1086/432798

[25] W. J. Munckhof, G. R. Nimmo, J. M. Schooneveldt, S. Schlebusch, A. J. Stephens, G. Williams, et al., "Nasal Carriage of Staphylococcus aureus, Including Community-Associated Methicillin-Resistant Strains, in Queensland Adults," Clinical Microbiology and Infection, Vol. 15, No. 2, 2009, pp. 149-155. doi:10.1111/j.1469-0691.2008.02652.x

[26] J. A. Severin, E. S. Lestari, K. Kuntaman, D. C. Melles,
M. Pastink, J. K. Peeters, et al., "Unusually High Prevalence of Panton-Valentine Leukocidin Genes among Methicillin-Sensitive Staphylococcus aureus Strains Carried in the Indonesian Population," Journal of Clinical Microbiology, Vol. 46, No. 6, 2008, pp. 1989-1995. doi:10.1128/JCM.01173-07

[27] K. O. Okon, P. Basset, A. Uba, J. Lin, B. Oyawoye, A. O. Shittu, et al., "Cooccurrence of Predominant PantonValentine Leukocidin-Positive Sequence Type (ST) 152 and Multidrug-Resistant ST 241 Staphylococcus aureus Clones in Nigerian Hospitals," Journal of Clinical Microbiology, Vol. 47, No. 9, 2009, pp. 3000-3003. doi:10.1128/JCM.01119-09

[28] F. Vandenesch, T. Naimi, M. C. Enright, G. Lina, G. R. Nimmo, H. Heffernan, et al., "Community-Acquired Methicillin-Resistant Staphylococcus aureus Carrying Panton-Valentine Leukocidin Genes: Worldwide Emergence," Emerging Infectious Diseases, Vol. 9, No. 8, 2003, pp. 978-984. doi:10.3201/eid0908.030089

[29] R. Ruimy, A. Maiga, L. Armand-Lefevre, I. Maiga, A. Diallo, A. K. Koumare, et al., "The Carriage Population of Staphylococcus aureus from Mali Is Composed of a Combination of Pandemic Clones and the Divergent Panton-Valentine Leukocidin-Positive Genotype ST152,' Journal of Bacteriology, Vol. 190, No. 11, 2008, pp. 3962-3968. doi:10.1128/JB.01947-07

[30] M. D. King, B. J. Humphrey, Y. F. Wang, E. V. Kourbatova, S. M. Ray and H. M. Blumberg, "Emergence of Community-Acquired Methicillin-Resistant Staphylococcus aureus USA 300 Clone as the Predominant Cause of Skin and Soft-Tissue Infections," Annals of Internal Medicine, Vol. 144, No. 5, 2006, pp. 309-317.

[31] B. Ghebremedhin, M. O. Olugbosi, A. M. Raji, F. Layer, R. A. Bakare, B. Konig, et al., "Emergence of a Community-Associated Methicillin-Resistant Staphylococcus aureus Strain with a Unique Resistance Profile in Southwest Nigeria," Journal of Clinical Microbiology, Vol. 47, No. 9, 2009, pp. 2975-2980. doi:10.1128/JCM.00648-09

[32] N. Ramdani-Bouguessa, M. Bes, H. Meugnier, F. Forey, M. E. Reverdy, G. Lina, et al., "Detection of MethicillinResistant Staphylococcus aureus Strains Resistant to Multiple Antibiotics and Carrying the Panton-Valentine Leukocidin Genes in an Algiers Hospital," Antimicrobial Agents and Chemotherapy, Vol. 50, No. 3, 2006, pp. 1083-1085.doi:10.1128/AAC.50.3.1083-1085.2006

[33] P. Dufour, Y. Gillet, M. Bes, G. Lina, F. Vandenesch, D. Floret, et al., "Community-Acquired Methicillin-Resistant Staphylococcus aureus Infections in France: Emergence of a Single Clone That Produces Panton-Valentine Leukocidin," Clinical Infectious Diseases, Vol. 35, No. 7, 2002, pp. 819-824. doi:10.1086/342576

[34] U. Seybold, E. V. Kourbatova, J. G. Johnson, S. J. Halvosa, Y. F. Wang, M. D. King, et al., "Emergence of Community-Associated Methicillin-Resistant Staphylococcus aureus USA300 Genotype as a Major Cause of Health Care-Associated Blood Stream Infections," Clinical Infectious Diseases, Vol. 42, No. 5, 2006, pp. 647-656. doi:10.1086/499815

[35] C. Sola, H. A. Saka, A. Vindel and J. L. Bocco, "High 
Frequency of Panton-Valentine Leukocidin Genes in Invasive Methicillin-Susceptible Staphylococcus aureus Strains and the Relationship with Methicillin-Resistant Staphylococcus aureus in Cordoba, Argentina," European Journal of Clinical Microbiology \& Infectious Diseases, Vol. 26, No. 4, 2007, pp. 281-286. doi:10.1007/s10096-007-0278-4

[36] F. C. Tenover, L. K. McDougal, R. V. Goering, G. Killgore, S. J. Projan, J. B. Patel, et al., "Characterization of a Strain of Community-Associated Methicillin-Resistant Staphylococcus aureus Widely Disseminated in the United States," Journal of Clinical Microbiology, Vol. 44, No. 1,
2006, pp. 108-118. doi:10.1128/JCM.44.1.108-118.2006

[37] A. Tong, S. Y. Tong, Y. Zhang, S. Lamlertthon, B. K. Sharma-Kuinkel, T. Rude, et al., "Panton-Valentine Leukocidin Is Not the Primary Determinant of Outcome for Staphylococcus aureus Skin Infections: Evaluation from the CANVAS Studies," PLoS One, Vol. 7, No. 5, 2012, e37212. doi:10.1371/journal.pone.0037212

[38] D. Weiskopf, B. Weinberger and B. Grubeck-Loebenstein, "The Aging of the Immune System," Transplant International, Vol. 22, No. 11, 2009, pp. 1041-1050. doi:10.1111/j.1432-2277.2009.00927.x 